\title{
Practice and exploration of 24-hour supervision model in personal protection supervision in a COVID-19 isolation ward
}

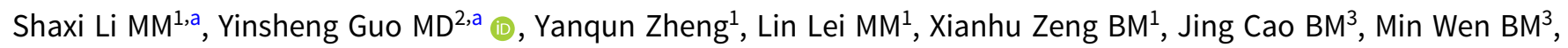 \\ Yingxia Liu MD and Ting Huang BM ${ }^{1}$ \\ ${ }^{1}$ Department of Healthcare-associated Infection Management, The Third People's Hospital of Shenzhen, Shenzhen, Guangdong, China, ${ }^{2}$ Environment and Health \\ Department, Shenzhen Center for Disease Control and Prevention, Shenzhen, Guangdong, China, ${ }^{3}$ Nursing Department, The Third People's Hospital of Shenzhen, \\ Shenzhen, Guangdong, China and ${ }^{4}$ Infectious Diseases Department, The Third People's Hospital of Shenzhen, Shenzhen, Guangdong, China
}

To the Editor-In 2020, the coronavirus disease 2019 (COVID-19) epidemic raged around the world. As of February 12, 1,716 medical staff from medical institutions throughout China had been confirmed as infected. ${ }^{1-3}$ Standard donning and doffing personal protective equipment (PPE) in isolation ward is crucial to reducing the risk of infection. A 24-hour supervision mode was applied in our hospital to maximize the prevention of nosocomial infection by finding and correcting mistakes in a timely way using continuous supervision.

\section{Methods}

From January 11 to February 26, 2020, a video observation mode was used to supervise the donning and doffing process of PPE. When entering the area, staff members did not take identification. Supervisors identified and recorded the errors and provided feedback after the process. Daily observation was conducted between 8:00 A.M. and 5:00 P.M. A 24-hour mode was implemented on February 27, and real-time intercom equipment was added. Identification information of HCWs was collected and a serial number was assigned. If mistakes were observed, they were recorded, and corrections were made in a timely way. Overall, 6 supervisors were assigned to a 24 -hour shift after unified training. The supervision contents of the 2 modes were consistent.

\section{Statistical analysis}

We used SPSS version 21.0 software (IBM, Armonk, NY) for statistical analyses. A $\chi^{2}$ test was used for statistical analysis among different groups, and logistic regression was used for multivariate analysis. $P<.05$ was considered statistically significant.

\section{Results}

In the video observation mode, 126 observations were recorded and 163 observations were recorded in the 24-hour mode. The PPE doffing procedures were carried out once for each person. In the video observation mode, the error rates of donning and doffing PPE were $2.38 \%$ and $12.70 \%$, respectively. The difference was statistically significant $\left(\chi^{2}=61.62 ; P<.001\right)$. In the 24 -hour mode, the error rates of donning and doffing PPE were $1.22 \%$ and $6.0 \%$,

Author for correspondence: Yingxia Liu, The Third People's Hospital of Shenzhen, No. 29, Bulan Road, Longgang District, Shenzhen, 510114, P.R. China. E-mail: yingxialiu@hotmail.com Or Ting Huang, E-mail address: hting622@hotmail.com.

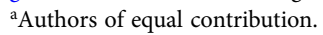

Cite this article: Li S, et al. (2021). Practice and exploration of 24-hour supervision model in personal protection supervision in a COVID-19 isolation ward. Infection Control \& Hospital Epidemiology, 42: 1027-1028, https://doi.org/10.1017/ice.2020.391 respectively. The difference was also statistically significant $\left(\chi^{2}=34.06, P<.001\right)$. In the 24 -hour mode, the total error rate of doffing PPE significantly decreased $\left(\chi^{2}=35.34, P<.001\right)$. In both modes, the main errors were related to wearing medical protective masks ( $7.14 \%$ vs $5.16 \%$ ) and hand hygiene ( $2.38 \%$ vs $1.24 \%)$. The main mistakes of doffing were related to the removal of disposable protective clothing $(32.54 \%$ vs $22.56 \%)$ and hand hygiene (30.16\% vs $19.85 \%)$.

We compared the error rates between the 2 groups and analyzed influencing factors in the 24-hour supervision mode. The error rate of HCWs aged $>40$ years was relatively high $(6.44 \%$, $\left.\chi^{2}=7.875 ; P=0.019\right)$. The error rate of HCWs was also relatively high $\left(11.52 \% ; \chi^{2}=26.94 ; P<.001\right)$. The main factor affecting the error rate was profession. Labor personnel were more likely to make mistakes than were medical staff (Table 1).

\section{Discussion}

In this investigation, the error rate was significantly higher in the PPE doffing procedure than in the PPE donning procedure, which is consistent with the research by Zhang et $\mathrm{al}^{4}$ during SARS. The processes of PPE donning and doffing are complicated, especially the doffing process, when the PPE may be contaminated. Therefore, the standardized performance of PPE removal is very important. ${ }^{5}$ After implementation of the 24-hour supervision mode, the error rates of all steps in donning and doffing PPE significantly decreased. One-to-one reminders and timely correction can prevent errors and strengthen the performance of HCWs. Furthermore, HCW awareness regarding risk of infection was raised considerably by these interventions.

During the overall PPE donning and doffing process, errors occurred most frequently while doffing the disposable protective clothing. Doffing action must be gentle, and extremes should be avoided to prevent personal as well as environmental contamination. Secondly, hand hygiene plays an important role in safe donning and doffing. In hand hygiene, the process and rubbing time are equally important. Before donning PPE, hand hygiene must be performed, and only clean hands can touch PPE. When doffing PPE, hand disinfection must be performed for each step and should be carried out again after all PPE has been removed.

In the 24-hour supervision mode, a higher error rate occurred among HCWs aged $>40$ years than in other age groups. However, no significant difference was detected in the error rate among different professional titles or genders associated with the full extent of training in our hospital in recent years. The major factor influencing the error rate, which is related to the lower education and lack of risk awareness, is the worker population. Nonmedical 
Table 1. Multifactor Logistic Regression Analysis of Error Rate

\begin{tabular}{|c|c|c|c|c|c|c|c|}
\hline \multirow[b]{2}{*}{ Factor } & \multirow{2}{*}{$\begin{array}{l}\text { Regression } \\
\text { Coefficient }\end{array}$} & \multirow{2}{*}{$\begin{array}{l}\text { Standard } \\
\text { Error }\end{array}$} & \multirow{2}{*}{$\begin{array}{l}\text { Wald } \\
\text { Value }\end{array}$} & \multirow[b]{2}{*}{$P$ Value } & \multirow[b]{2}{*}{$\operatorname{Exp}(B)$} & \multicolumn{2}{|c|}{$95 \%$ Confidence Interval } \\
\hline & & & & & & Upper & Lower \\
\hline Profession & 1.689 & 0.799 & 4.473 & 0.034 & 5.413 & 1.132 & 25.891 \\
\hline
\end{tabular}

HCW s are considered a high-risk group for occupational exposure. Improving their risk and self-protection awareness and developing a standard of safe behavior are key issues in future work.

This investigation also has several limitations. The investigation was conducted after the peak period of our domestic epidemic, when the error rate might have been relatively lower than previously. The on-site supervision, video supervision, and intercom reminders were innovatively combined in the 24-hour supervision mode. In addition, the continuous mode provides 24-hour supervision, reminders, and thus, protection for medical personnel. This continuity helps these workers regulate their behavior and reduce risk. Overall, this intervention has proven informative, effective, and successful.

Acknowledgments. The authors would like to thank all the participants in our study.

Financial support. This study was supported by grants from the Science and Technology Innovation Committee of Shenzhen Municipality (grant no. 202002073000001), the National Key Research and Development Program (grant no. 2020YFC0841700), the National Science Foundation of China (grant no. 81773395), and the Sanming Project of Medicine in Shenzhen (grant no. SZSM201811070).

Conflicts of interest. All authors report no conflicts of interest relevant to this article.

\section{References}

1. Epidemiology Working Group for NCIP Epidemic Response Chinese Center for Disease Control and Prevention. The epidemiological characteristics of an outbreak of 2019 novel coronavirus diseases (COVID-19) in China. Zhonghua liu xing bing xue za zhi = Zhonghua liuxingbingxue zazhi 2020;41:145-151.

2. Honda $\mathrm{H}$, Iwata K. Personal protective equipment and improving compliance among healthcare workers in high-risk settings. Curr Opin Infect Dis 2016;29:400-406.

3. Wang C, Horby PW, Hayden FG, Gao GF. A novel coronavirus outbreak of global health concern. Lancet 2020;395:470-473.

4. Guan WJ, Ni ZY, Hu Y, et al. Clinical characteristics of coronavirus disease 2019 in China. New Engl J Med 2020;382:1708-1720.

5. Wang D, Hu B, Hu C, et al. Clinical characteristics of 138 hospitalized patients with 2019 novel coronavirus-infected pneumonia in Wuhan, China. JAMA 2020;323:1061-1069.

\title{
Frequency of facial touching in patients with suspected COVID-19 during their time in the waiting room
}

\author{
Eduardo Perez-Alba MD ${ }^{1}$ (1) Laura Nuzzolo-Shihadeh $\mathrm{MD}^{1}$ (1), Alejandro Fonseca-Ruiz MD² (1),

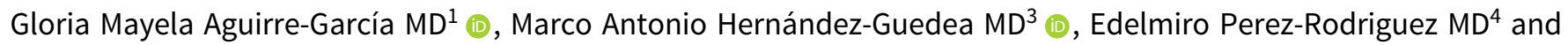 \\ Adrián Camacho-Ortiz MD² (1) \\ ${ }^{1}$ Servicio de infectología, Hospital Universitario Dr. José Eleuterio González, Universidad Autónoma de Nuevo León, Monterrey, Mexico, ²Departamento de \\ Epidemiología Hospitalaria y Servicio de Infectología, Hospital Universitario Dr. José Eleuterio González, Universidad Autónoma de Nuevo León, Monterrey, \\ Mexico, ${ }^{3}$ Subdirección de Asistencia Hospitalaria, Hospital Universitario Dr. José Eleuterio González, Universidad Autónoma de Nuevo León, Monterrey, Mexico \\ and ${ }^{4}$ Dirección Hospitalaria, Hospital Universitario Dr. José Eleuterio González, Universidad Autónoma de Nuevo León, Monterrey, Mexico
}

To the Editor-Coronavirus disease 2019 (COVID-19) is a novel disease caused by severe acute respiratory coronavirus virus 2 (SARS-CoV-2), which has spread worldwide. ${ }^{1}$ Viral transmission is suspected to occur through droplets produced predominantly while coughing and sneezing. ${ }^{2}$ Alternatively, viral particles may remain infectious in inert surfaces and act as fomites. ${ }^{3}$

Although transmission by droplet aspiration and contact with other respiratory secretions are well described as contagion mechanisms, face touching has not been as extensively discussed. ${ }^{4-6}$ As universal masking gains popularity among healthcare

\footnotetext{
Author for correspondence: Adrian Camacho-Ortiz, E-mail: acamacho_md@yahoo. com

Cite this article: Perez-Alba E, et al. (2021). Frequency of facial touching in patients with suspected COVID-19 during their time in the waiting room. Infection Control \& Hospital Epidemiology, 42: 1028-1030, https://doi.org/10.1017/ice.2020.389
}

professionals (HCPs), the fact that they promote face touching must not be forgotten. ${ }^{7}$ Despite the latter, face masks have a crucial role in protection, but whether they provide protection for patients in the outpatient setting is unknown.

This phenomenon could be crucial in the transmission of SARS-CoV-; thus, we explored the frequency of face touching in patients with possible COVID-19 awaiting evaluation in an ambulatory clinic.

\section{Methods}

We designed and implemented a study in which video cameras were installed in the waiting room of a respiratory infection diagnosis unit during March 2020. As patients waited for care their behavior was recorded and later logged. Upon arrival to the clinic, 